\section{Aneurisma coronario secundario a enfermedad de Kawasaki en paciente adulto. Caso Clínico}

\author{
MILTON ALCAÍNO I. ${ }^{1}$, DENISSE LAMA H. ${ }^{2}$, \\ GONZALO PEREIRA R. ${ }^{2}$
}

\section{Coronary aneurysm secondary to Kawasaki disease in an adult. Report of one case}

\begin{abstract}
Kawasaki disease $(K D)$ can be a primary cause of an acute coronary syndrome in young adults, with or without a history of KD during childhood. We report a 31 year old female, admitted for acute chest pain. She had negative T waves on DIII and AVF leads of the electrocardiogram. Troponins were positive. A cardiac magnetic resonance was compatible with an acute inferior myocardial infarction. In the coronary angiography she had a calcified complete occlusion of the proximal right coronary artery. The left coronary artery had no visible lesions. A computed angiogram showed an extensive calcified and thrombotic aneurysm of the right coronary artery, compatible with Kawasaki disease (KD). The patient was discharged seven days after admission in good conditions.

(Rev Med Chile 2021; 149: 803-806)

Key words: Aneurysm; Coronary Vessels; Mucocutaneous Lymph Node Syndrome.
\end{abstract}

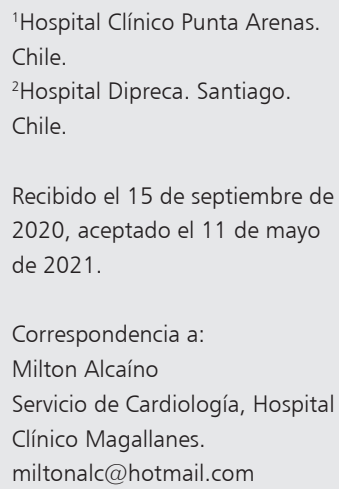

A unque la frecuencia de los SCA en pacientes jóvenes es difícil de establecer, se estima que 4 a $10 \%$ se producen en pacientes menores de 40 a 45 años. La primera causa es la enfermedad coronaria ateroesclerótica y los factores de riesgo habituales son asimismo importantes, también el uso de drogas ilícitas juega un rol en este grupo de pacientes. Existen otras causas: disección coronaria, vasoespasmo, embolias, Takotsubo, compromiso microvascular, y vasculitis o secuela de vasculitis, como la EK en paciente adulto ${ }^{1,2}$. Presentamos el caso de una paciente que consultó en el Hospital Dipreca por dolor torácico, interpretado inicialmente como de otra etiología, y que, analizado su cuadro clínico, junto a los exámenes efectuados, se concluyó que se trataba de una EK. Esta publicación de este caso cuenta con consentimiento de la paciente.

\section{Caso clínico}

Paciente de 31 años, sin antecedentes mórbidos de relevancia, ni factores de riesgo cardiovascular. Consulta por haber presentado dolor retroesternal de reposo de 16 horas de evolución. Asintomática en el momento del ingreso, presión arterial 125/70, pulso 74 latidos por minuto, afebril, auscultación cardiaca normal, sin signos de congestión pulmonar ni otros hallazgos de relevancia al examen físico. En el electrocardiograma existía inversión de T en DIII y AVF. Troponina I 6,77 ng/ml. CK total 593 U/lt. MB 79 U/lt. Hematocrito 39\%. Leucocitos $9.400 \mathrm{~mm}^{3}$. Fórmula normal. Plaquetas normales. VHS 10 $\mathrm{mm} / \mathrm{h}$. Debido a la edad de la paciente y ausencia de factores de riesgo, se solicitó una resonancia magnética cardíaca, que evidenció edema de la pared inferior basal transmural, aquinesia de 
ese segmento y un patrón de realce tardío con gadolinio, compatible con un infarto de pared inferior. Se inicia tratamiento con ácido acetilsalicílico, clopidogrel, nitroglicerina y estatinas. Se efectúa posteriormente una coronariografía, que demuestra una oclusión a nivel del tercio proximal de la ACD, con imágenes de calcificación en los bordes. Los otros vasos coronarios no tenían lesiones angiográficamente significativas. El AngioTAC coronario, reveló un extenso aneurisma trombosado de la $\mathrm{ACD}$, comprometiendo el tercio proximal, medio y distal, de $9,8 \mathrm{~cm}$ de longitud y $9 \mathrm{~mm}$ de diámetro, con calcificaciones parietales en el tercio proximal y medio (Figuras la y 1b), compatible por sus características con EK. La ACI no tenía lesiones demostrables (Figuras $2 \mathrm{a}$ y $2 \mathrm{~b}$ ). La paciente evoluciona satisfactoriamente, siendo dada de alta a los 7 días con ácido acetilsalicílico, acenocumarol, estatinas y $\beta$-bloqueadores. Posteriormente, debido a angina de esfuerzos, se inició
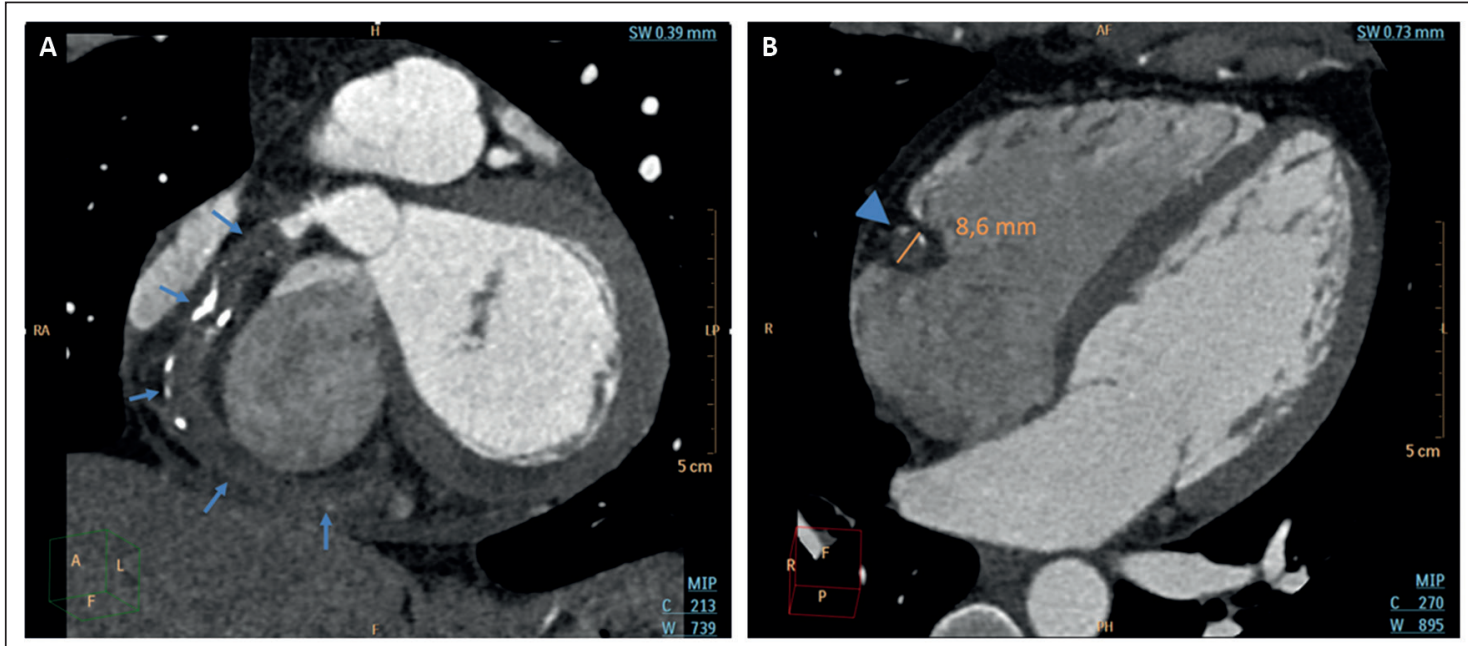

Figura 1. Aneurisma de la arteria coronaria derecha (CD). A: Reconstrucción coronal del trayecto de la coronaria derecha (flechas) que muestra su dilatación, trombosis y calcificaciones parietales. B: Reconstrucción en 4 cámaras con el aneurisma en el tercio medio de la CD (cabeza de flecha).
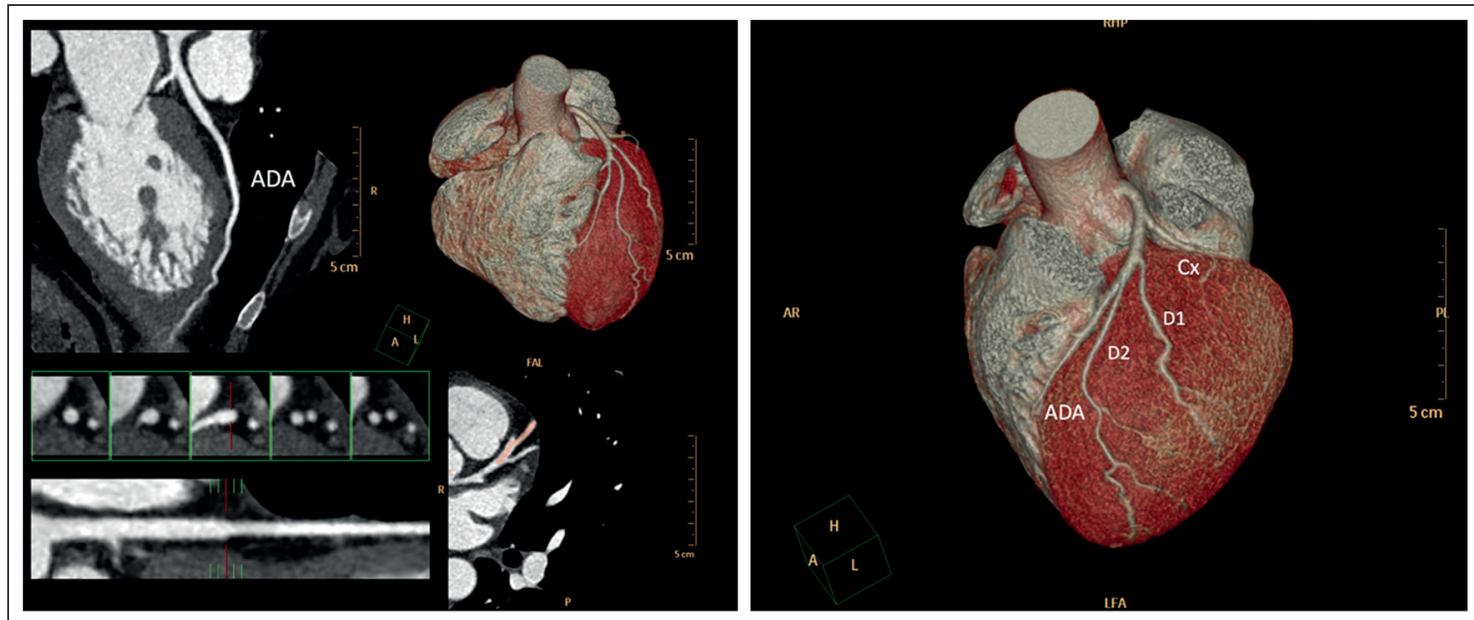

Figura 2. Arteria coronaria izquierda. A: Reconstrucción curva de la arteria descendente anterior (ADA) que demuestra su calibre y permeabilidad hasta la región distal. B: Reconstrucción VRT de la arteria descendente anterior, diagonales (D1 y D2) y circunfleja proximal (Cx). 
un plan de rehabilitación cardíaca, con buen resultado, encontrándose actualmente asintomática.

\section{Discusión}

La EK es una vasculitis aguda sistémica autolimitada, que afecta a las arterias de mediano calibre, con predilección por las arterias coronarias. Comienza como un cuadro febril, compromiso muco-cutáneo y adenopatías cervicales ${ }^{3}$. Afecta predominantemente a niños menores de 5 años y especialmente entre los 6 y 11 meses de edad, con cierta predominancia en el sexo masculino. Es más frecuente en Japón, con una incidencia en ese país de 217 a 265 casos al año por 100.000 en niños menores de 5 años. También es más frecuente en los estados de California y Hawaii, por la mayor frecuencia de descendientes de la zona Asia Pacífico ${ }^{4}$. La etiología de la EK es desconocida. Consiste en una respuesta inmune a un agente desconocido, posiblemente infeccioso o algún antígeno del medio ambiente. Respecto al compromiso arterial, en la fase inflamatoria aguda, los neutrófilos inicialmente invaden la pared arterial, posteriormente predominan los linfocitos $\mathrm{T}$, especialmente linfocitos CD8, células dendríticas, monocitos, macrófagos y células plasmáticas. Los macrófagos, linfocitos $\mathrm{T}$ y los fibroblastos, que también comienzan a aparecer, secretan factores de crecimiento del endotelio vascular, metaloproteinasas, otras citoquinas e interleuquinas, que destruyen la lámina elástica interna y externa produciéndose dilatación y aneurismas ${ }^{5-6}$. Con las semanas, las células inflamatorias son reemplazadas por fibroblastos y monocitos produciéndose un engrosamiento fibrointimal, con trombosis y estenosis. Puede presentarse un SCA, muerte por infarto del miocardio o por ruptura de los aneurismas.

El diagnóstico de la EK es clínico. Se basa en la presencia de fiebre por al menos 5 días y con al menos 4 de los siguientes criterios:

- Eritema y lesiones en los labios, lengua de frambuesa y/o eritema de la mucosa oral y faríngea.

- Compromiso bilateral conjuntival con exudado.

- Rush macopapular, eritroderma difuso, o eritema similar al multiforme.

- Eritema y edema de las manos y pies en la fase aguda o descamación periungeal en la fase subaguda.
- Adenopatías cervicales mayores o iguales a $1,5 \mathrm{~cm}$ generalmente unilateral.

El diagnóstico también puede efectuarse con menos criterios, si existe suficiente experiencia y especialmente si se detectan aneurismas coronarios. La EK también puede tener otras manifestaciones cardiacas, como miocarditis, pericarditis, compromiso valvular, especialmente mitral o compromiso de la aorta ascendente. También puede haber compromiso respiratorio, neurológico, digestivo o genitourinario. El tratamiento se basa en el uso de inmunoglobulina endovenosa y ácido acetilsalicílico ${ }^{7}$.

La presencia de aneurismas puede ser de hasta $25 \%$, en pacientes no tratados y aun con tratamiento, existe un porcentaje de pacientes que persistirá con esta patología hasta la edad adulta. En Japón, hasta 9,0\% de los infartos del miocardio y muertes súbitas en adultos jóvenes, pueden ser por EK. En varios de estos casos, no hubo diagnóstico en la infancia, porque presentaron un cuadro incompleto, se realizó otro diagnóstico o el cuadro no fue detectado ${ }^{8,9}$, como fue el caso de esta paciente, que no relató haber tenido un cuadro compatible con EK en su infancia.

En los pacientes que desarrollan aneurismas, la proliferación neointimal produce una seudonormalización del lumen, asociándose a trombos, calcificación y estenosis, especialmente en aneurismas mayores de 6 a $8 \mathrm{~mm}$ de diámetro ${ }^{6,7,9}$. Como consecuencia de estos fenómenos se produce isquemia, los pacientes pueden tener angina, SCA, arritmias graves y/o muerte. En un adulto joven, que se presenta con un SCA, se debe considerar la EK como una causa, especialmente si en el estudio de las arterias coronarias se encuentran aneurisma o aneurismas, los cuales pueden estar calcificados, más aún si no hay factores de riesgo y el resto del árbol coronario no tiene lesiones angiográficamente demostrables ${ }^{10}$.

Esta paciente, la cual no tenia factores de riesgo cardiovascular, tenia un aneurisma de $9 \mathrm{~mm}$. de diámetro, calcificado, remodelado y con el segmento arterial distal al aneurisma, así como el resto de los vasos coronarios, angiográficamente normales, lo cual es diferente de una patología ateroesclerótica. En esta patología el compromiso es más difuso y en pacientes jóvenes, muchas veces sin calcificaciónes, lo cual es habitual en los aneurismas de la $\mathrm{EK}^{10}$. El aneurisma, en este 
caso, por su diámetro tenía riesgo de oclusión y provoco manifestaciones clínicas de isquemia, aunque existen casos de oclusión asintomática. Esta paciente no tenía compromiso de la función sistólica del ventrículo izquierdo, insuficiencia mitral, ni dilatación significativa de la aorta ascendente, lo cual también se observa como secuela de la $\mathrm{EK}^{9}$. No se realizó estudio de la función endotelial, pero está documentado que se encuentra alterada en pacientes con aneurismas en la $\mathrm{EK}^{10}$. Respecto al tratamiento y seguimiento, las guías de la Sociedad Americana de Cardiología, como las guías japonesas, no son claras respecto a el manejo con antiplaquetarios o anticoagulación en casos con aneurismas de estas características ocluidos $^{7-11}$. Las recomendaciones son para pacientes con aneurismas no ocluidos, considerando el tamaño del aneurisma y presencia de síntomas o signos de isquemia. Las anteriores guías de la Asociación Americana de Cardiología consideran el tratamiento para casos como esta paciente, con el uso de ácido acetilsalicílico, anticoagulación y B- bloqueo ${ }^{12}$. También, al existir disfunción endotelial, las nuevas guías recomiendan estatinas y eventualmente inhibidores de la enzima convertidora ${ }^{7}$. La recomendación de control es cada 6 meses con ecocardiograma y ECG, además de pruebas de isquemia anual.

Finalmente, a raíz de la pandemia provocada por el virus SARS-CoV-2, se ha descrito en niños $y$ adolescentes, una enfermedad que tiene varias características similares a la EK, pero también a veces con manifestaciones mas amplias y diferentes, con mayor frecuencia de compromiso cardiovascular y también con desarrollo de aneurismas coronarios con una frecuencia de 8 a 20\% ${ }^{13,14}$. Aun se requiere mayor conocimiento y seguimiento de estos pacientes. Sin embargo, es muy posible que a futuro se observen casos similares al reportado, en pacientes que hubieran presentado esta nueva patología.

\section{Conclusión}

En un paciente joven con un SCA, especialmente sin factores de riesgo y ante el hallazgo de aneurisma o aneurismas en las arterias coronarias, se debe considerar como etiología la EK, mas aun si tienen calcificación y el resto del árbol coronario es angiográficamente normal.

\section{Referencias}

1. Azar R, Verheugt F, Saperia G. Coronary Heart Disease and Myocardial Infarction in Young Adults. uptodate. com. Mar 14, 2020.

2. Gulati R, Behfar A, Narula J, Kanwar A, Lerman A, Cooper L, et al. Acute Myocardial Infarction in Young Adults. Mayo Clin Proc. 2020; 95: 136-56.

3. Kato H, Koike S, Yamamoto M, Ito Y, Yano E. Coronary Aneurysm in Infants and Young Children with Acute Febrile Mucocutaneous Lymph Node Syndrome. J Pediatr. 1975; 86: 892-8.

4. Uehara R, Belay E. Epidemiology of Kawasaki Disease in Asia, Europe, and the United States. J Epidemiol. 2012; 22: 79-85.

5. Rowley A, Shulman S. Pathogenesis and Management of Kawasaki Disease. Expert Rev Anti Infect Ther. 2010; 2: 197-203.

6. Newburger J, Takahashi M, Burns J. Kawasaki Disease. J Am Coll Cardiol. 2016; 67: 1738-49.

7. McCrindle B, Rowley A, Newburger J, Burns J, Bolger A, Gewitz M, et al. Diagnosis, Treatment and LongTerm Management of Kawasaki Disease: A Scientific Statement for Health Professionals From the American Heart Association. Circulation 2017; 135: e927-e999.

8. Daniels L, Tjajadi M, Walford H, Jiménez-Fernández S, Trofimenko V, Fick D, et al. Prevalence of Kawasaki Disease in Young adults with Suspected Myocardial Ischemia. Circulation 2012; 125: 2447-53.

9. Denby K, Clark D, Markham L. Managament of Kawasaki Disease in Adults. Heart 2017; 103: 1760-69.

10. Gordon J, Kahn A, Burns J. When Children with Kawasaki Disease Grow Up. J Am Coll Cardiol. 2009; 54: 1911-20.

11. JSC Joint Working Group. Guidelines for Diagnosis and Management of Cardiovascular Sequelae in Kawasaki Disease. Circ J. 2014; 78: 2521-62.

12. Newburger J, Takahashi M, Gerber M, Gewitz M, Tani L, Burns J, et al. Diagnosis, Treatment and Long-Term Management of Kawasaki Disease: A Statement for Health Professionals from The Committee on Rheumatic Fever, Endocarditis and Kawasaki Disease, Council on Cardiovascular Disease in the Young, American Heart Association. Circulation 2004; 110: 2747-71.

13. Feldstein L, Rose E, Horwitz S, Collins J, for the Overcoming COVID-19 Investigators, and the CDC COVID-19 Response Team. Multisystem Inflammatory Syndrome in U.S. Children and Adolescents. N Engl J Med. 2020; 383: 334-46.

14. Levin M. Childhood Multisystem Inflammatory Syndrome - A New Challenge in the Pandemic. N Engl J Med. 2020; 383: 393-5. 